\title{
A Contextual Planck Parameter and the Classical Limit in Quantum Cosmology
}

\author{
John D. Barrow ${ }^{1}$ · João Magueijo ${ }^{2}$
}

Received: 28 July 2020 / Accepted: 30 January 2021 / Published online: 19 February 2021

(C) The Author(s) 2021

\begin{abstract}
We propose that whatever quantity controls the Heisenberg uncertainty relations (for a given complementary pair of observables) it should be identified with an effective Planck parameter. With this definition it is not difficult to find examples where the Planck parameter depends on the region under study, varies in time, and even depends on which pair of observables one focuses on. In quantum cosmology the effective Planck parameter depends on the size of the comoving region under study, and so depends on that chosen region and on time. With this criterion, the classical limit is expected, not for regions larger than the Planck length, $l_{P}$, but for those larger than $l_{Q}=\left(l_{P}^{2} H^{-1}\right)^{1 / 3}$, where $H$ is the Hubble parameter. In theories where the cosmological constant is dynamical, it is possible for the latter to remain quantum even in contexts where everything else is deemed classical. These results are derived from standard quantization methods, but we also include more speculative cases where ad hoc Planck parameters scale differently with the length scale under observation. Even more speculatively, we examine the possibility that similar complementary concepts affect thermodynamical variables, such as the temperature and the entropy of a black hole.
\end{abstract}

\section{Introduction}

Although promoting the constants of Nature to dynamic fields is hardly a novelty, structural fundamental constants, such as $c$ and $\hbar$, are usually spared this transformation. In particular, Planck's constant (with notable exceptions, for example [1-4]) has had its constancy left largely unchallenged. This is perhaps less warranted by physics conservatism than one might think. The true hallmark of quantum behaviour

João Magueijo

j.magueijo@imperial.ac.uk

1 DAMTP, Centre for Mathematical Sciences, Cambridge University, Wilberforce Rd., Cambridge CB3 0WA, UK

2 Theoretical Physics Group, The Blackett Laboratory, Imperial College, Prince Consort Rd., London SW7 2BZ, UK 
is the impossibility of jointly measuring complementary observables, usually (but not always) resulting from their non-commutativity and associated Heisenberg uncertainty relations [5-8]. Whatever function controls these relations (or the commutator) acts as an effective Planck parameter, even if it is not a constant ${ }^{1}$, and even if $\hbar$ is only one of its contributing factors, as we shall find in concrete examples in this paper. Hence, it is possible that the effective Planck parameter has a variability induced by context and circumstance, depending on the system under study and the chosen pair of variables, and possibly evolving in time for a given system and pair.

In this paper we make this point in a variety of situations. In Sect. 2 we consider standard quantum cosmology, but rather than extending the action to a whole closed manifold, we consider the context of fixed comoving spatial regions. The effective Planck parameter is then found to depend on the size of the comoving region under study, and so depends on the chosen region and on time. By studying this parameter we discover that the classical limit is expected, not for regions larger than the Planck length, $l_{P}$, but for those larger than a mesoscopic scale, of the order of the proton scale.

In Sect. 3 we extend our considerations to theories for which the cosmological constant, $\Lambda$, is dynamical, with the surprising result that it is possible for $\Lambda$ to remain quantum even in contexts where everything else is deemed classical. Finally in Sect. 4 we speculate on cases where ad hoc Planck parameters scale differently with the length scale under observation.

We conclude with a general discussion of the implications of our findings.

\section{Planck's Parameter in Standard Quantum Cosmology}

Let us consider the minisuperspace (MSS) quantum cosmology (QC) following from the Einstein-Hilbert action. The reduced action can be written as:

$$
S=6 \kappa V_{c} \int d t\left(a^{2} \dot{b}+N a\left(b^{2}+k-\frac{\Lambda}{3} a^{2}\right)\right),
$$

where $\kappa=1 /\left(16 \pi G_{N}\right)$ (with $G_{N}$ Newton's constant), $a$ is the expansion factor, $b$ is the expansion rate (with $b=\dot{a} / N$ on-shell), $N$ is the lapse function associated with time coordinate $t$ and $k=0, \pm 1$ is the normalized spatial curvature [9, 10]. Crucially, for the purpose of this paper,

$$
V_{c}=\int d^{3} x \sqrt{h}
$$

is an integral in comoving spatial variables over the region under study (assumed to be fixed in comoving variables) and $h$ is the determinant of the comoving (timeindependent) 3-metric. Usually in QC, one sets $k=1$ and integrates over the whole sphere, so that $V_{c}=2 \pi^{2}$. In some cases, one considers topologically non-trivial

\footnotetext{
${ }^{1}$ We will adopt the term "parameter" for this reason.
} 
compact spaces with $k=0,-1$. Here, we propose that the region under study be a generic fixed region in comoving coordinates, where homogeneity and isotropy can be assumed. Different such regions may, or not, have different properties, so this partitioning can be at a scale different from the scale of homogeneity. It can also be a smoothing scale, if strict homogeneity is not assumed. Whatever the interpretation, we assume that the different regions do not interact. The overall wave-function, therefore, is the cross-product of the wave-functions for different regions, each with an associated $V_{c}$ :

$$
\psi=\bigotimes_{i} \psi_{i}\left(a ; k, V_{c i}\right)
$$

Underlying this ansatz is the assumption that some degrees of freedom (such as the graviton's) are frozen or are ignorable. Nevertheless, the integration over the spatial degrees of freedom leaves a trace, in the form of $V_{c i}$, in the pre-factor of the classical action (1), and in the quantum wave-function for each of these regions.

In the absence of matter other than $\Lambda$, the pre-factors in (1) are irrelevant for the classical theory. But they are relevant for the quantum theory, and indeed they contribute to the effective $\hbar$. They propagate into the Poisson Bracket (PB):

$$
\left\{b, a^{2}\right\}=\frac{1}{6 \kappa V_{c}},
$$

and upon quantization into the commutator:

$$
\left[\hat{b}, \hat{a^{2}}\right]=\frac{i l_{P}^{2}}{3 V_{c}}
$$

where $l_{P}=\sqrt{8 \pi G_{N} \hbar}$ is the reduced Planck length.

Obviously, we could normalize the phase space so that the PB is 1 , but if we insist on working with observables that become our favoured classical variables when classical cosmology emerges, then the function controlling their commutator is a variable depending on contextual factors, such as the volume of the region under study, and may vary in time. For example, we could have worked with a conjugate to $a^{2}$ given by $\Pi_{a}^{2}=6 \kappa V_{c} b$, i.e. a version of the Hubble parameter $H$ multiplied by the volume under study. But this is not our "favoured" variable: there is no point in multiplying what comes out of the Hubble diagram by $V_{c}$. Within MSS the observable is simply $H$ (or $b$ ), but then the factor of $1 / V_{c}$ on the right hand side (5) is unavoidable. This factor is not arbitrary. It is determined by the action, and by the choice of relevant observable, which in many cases (including this one) is obvious, given the MSS approximation. And yet, this factor has practical consequences, since it affects the quantum fluctuations and uncertainty relations. It says that these depend on whether we look at the whole Universe, the current Hubble volume, or just the local cluster, as we now explain further.

There is some controversy over the details of the mechanism leading to classicality (and even the definition of the latter; see, e.g. [11-13]); but in view of (5), 
whatever brings about the classical limit in quantum cosmology, the process is controlled by the effective Planck parameter:

$$
\mathfrak{h}=\frac{l_{P}^{2}}{3 V_{c}} .
$$

This contains $\hbar$, but also $G_{N}$ and $V_{c}$, so the classical limit depends on the size of the region under study. Given the presence of $G_{N}$ (and so $l_{P}$ ) in the commutator, one might expect that any region with a physical size larger than the Planck length is classical, but that is not what the Heisenberg relations tell us. These can be derived purely kinematically ${ }^{2}$, and result in [6]:

$$
\sigma(b) \sigma\left(a^{2}\right) \geq \frac{\mathfrak{h}}{2}
$$

from which a dimensionless version can be written:

$$
\frac{\sigma(b)}{\langle b\rangle} \frac{\sigma\left(a^{2}\right)}{\left\langle a^{2}\right\rangle} \geq \frac{\mathfrak{h}_{1}^{\prime}}{2},
$$

with

$$
\mathfrak{h}_{1}^{\prime}=\frac{l_{P}^{2}}{3 V_{c}\left\langle a^{2}\right\rangle\langle b\rangle} .
$$

This contextual Planck parameter is dimensionless and depending on whether it is much larger than 1 , or the opposite, the system may be declared quantum or classical. It depends not only on the comoving volume of the region under study, but also on time, as the physical size of the region increases (via the scale factor $\left\langle a^{2}\right\rangle$ ) and the Hubble scale, implicit in $\langle b\rangle$, decreases.

For regions and times for which $\sigma(b) \ll\langle b\rangle$ and $\sigma\left(a^{2}\right) \ll\left\langle a^{2}\right\rangle$, we may approximate

$$
\mathfrak{h}_{1}^{\prime} \approx \frac{l_{P}^{2}}{3\langle V\rangle\langle H\rangle},
$$

where $V$ is the proper volume of the comoving region under study (i.e.: $V=V_{c} a^{3}$ ) and $H=b / a$ is the Hubble parameter. In this simple model, the criterion for quantum space-time fluctuations to occur is not that one looks at length scales smaller than the Planck scale, but smaller than the scale:

$$
l_{Q} \sim\left(l_{P}^{2} H^{-1}\right)^{1 / 3} .
$$

This scale increases in time, as $H$ decreases. Nowadays, it is bigger than the Planck scale, but still microphysical (and hence one may declare the Friedman metric

\footnotetext{
${ }^{2}$ I.e.: from the commutator alone, without using the Hamiltonian or the dynamics.
} 
inapplicable). But taking this calculation as a toy model, the point remains that the relevant scale is not $10^{19} \mathrm{GeV}$, but around $0.1 \mathrm{GeV}$. Indeed it is a time-dependent scale, related to the time-dependent Planck parameter $\mathfrak{h}_{1}^{\prime 3}$

Note that within the approximation leading to (10) we can also take the average of the Hamiltonian constraint:

$$
\mathcal{H}=b^{2}+k-\frac{\Lambda}{3} a^{2}=0
$$

associated with some Lagrange multiplier, $N$. Since, in the same approximation, $\left\langle b^{2}\right\rangle \approx\langle b\rangle^{2}$ and $\left\langle a^{3}\right\rangle \approx\langle a\rangle^{3} \approx\left\langle a^{2}\right\rangle^{3 / 2}$, this leads to:

$$
\mathfrak{h}_{1}^{\prime} \approx \frac{l_{P}^{2}}{3\langle V\rangle \sqrt{\frac{\Lambda}{3}-\frac{k}{\langle a\rangle^{2}}}} .
$$

Obviously, these assumptions already assume that we are in a regime where $\mathfrak{h}_{1}^{\prime} \ll 1$. We could also include a matter term $\rho$ in the Hamiltonian constraint and this would appear in (13).

\section{Models with a Dynamical Cosmological Constant}

Other models may be considered, for example a version of Einstein-Cartan theory including a quasi-Euler term, where $\Lambda$ becomes dynamical $[14,15]$. The Hubble variable $b$ is then identified with the parity-even part of the connection, and on-shell $b=\dot{a}+T a$, where $T$ is the parity-even component of the torsion field. A parity-odd component of the connection and torsion may be present (this is the so-called Cartan spiral staircase [16]), but we shall set it to zero here, and confine ourselves to the parity-even branch of the phase space $[14,15,17]$.

Such a theory is interesting for this paper because $\Lambda$, by virtue of classically being part the phase space, upon quantization becomes an observable subject to uncertainty relations. Besides the Hamiltonian constraint, the theory has a constraint forcing the momentum $\Pi$ conjugate to $\Lambda^{-1}$ to be proportional to the Chern-Simons (CS) integral. Spelling it out $[17]^{4}$, the action (1) is extended to:

\footnotetext{
${ }^{3}$ After this paper was submitted, the work of [21] was brought to our attention. The "mesoscopic" scale derived in that reference is the same as ours, for the same reason (which is essentially of dimensional analysis). Whilst our result is more general (it does not depend on the concrete dynamics) the work of [21], precisely for being less general, is more concrete, and even derives wave functions for the Universe incorporating this scale.

${ }^{4}$ With some cosmetic modifications with respect to [17], namely $\Pi \rightarrow 2 \Pi$, and $V \rightarrow 6 V$. This prevents the appearance of effective Planck parameters that differ merely by numerical factors.
} 


$$
\begin{aligned}
S= & 6 \kappa V_{c} \int d t\left(a^{2} \dot{b}+\Pi \frac{d \Lambda^{-1}}{d t}\right. \\
& +N a\left(b^{2}+k-c^{2}-\frac{\Lambda}{3} a^{2}\right) \\
& \left.+V\left(\Pi-\left(b^{3}+3 b\left(k-c^{2}\right)\right)\right)\right),
\end{aligned}
$$

so that the PBs are now (4) and,

$$
\left\{\Lambda^{-1}, \Pi\right\}=\frac{1}{6 \kappa V_{c}},
$$

with a new constraint, associated with the Lagrange multiplier $V$, given by:

$$
\mathcal{V}=\Pi-\tau_{C S}=0
$$

where :

$$
\tau_{C S}=b^{3}+3 b k
$$

It can be shown [10] that this is the imaginary part of the CS functional in the MSS approximation, stripped of factors arising from the spatial integration. This quantity has been proposed as a measure of time in quantum gravity [18], the so-called Chern-Simons time.

Since the constraints are first class [17], the PBs imply the commutation relation (5) as well as:

$$
\left[\hat{\Lambda}^{-1}, \hat{\Pi}\right]=\frac{i l_{P}^{2}}{3 V_{c}}=i \mathfrak{h} .
$$

From these, Heisenberg relations and their effective Planck parameters may be derived. In addition to (7), which is still valid, we have:

$$
\sigma\left(\Lambda^{-1}\right) \sigma\left(\tau_{C S}\right) \geq \frac{\mathfrak{h}}{2}
$$

from which a dimensionless version ${ }^{5}$ can be derived:

$$
\frac{\sigma\left(\Lambda^{-1}\right)}{\left\langle\Lambda^{-1}\right\rangle} \frac{\sigma\left(\tau_{C S}\right)}{\left\langle\tau_{C S}\right\rangle} \geq \frac{\mathfrak{h}_{2}^{\prime}}{2}
$$

with

\footnotetext{
${ }_{5}^{5}$ We stress that all dimensionless relations we have written respect the symmetries of the underlying variables. For example, if $k=0$, then $a$ can be multiplied by any constant, but this drops out of the dimensionless relations. The analogy with $x$ and $p$, where dividing by $\langle x\rangle$ would clash with the translation invariance, therefore does not apply. None of the variables in our relations has shift invariance.
} 


$$
\mathfrak{h}_{2}^{\prime}=\frac{l_{P}^{2}}{3 V_{c}\left\langle\tau_{C S}\right\rangle\left\langle\Lambda^{-1}\right\rangle}
$$

This provides us with an example where the same system may have different effective Planck parameters for different pairs of variables. These may be wildly different. For example, in the quantum domain (where $\mathfrak{h}_{i}^{\prime}$ are much larger than 1), we could conjure a state with very different $\left\langle a^{2}\right\rangle\langle b\rangle$ and $\left\langle\tau_{C S}\right\rangle\left\langle\Lambda^{-1}\right\rangle$, so that $\mathfrak{h}_{1}^{\prime}$ and $\mathfrak{h}_{2}^{\prime}$ differ appreciably. The construction of coherent states, saturating the bound in such an unusual situation, will undoubtedly be interesting. One can even envisage hybrid situations, where the system has gone classical for one pair of variables, while remaining quantum for another pair. We should stress that most of the conditions for classicality we wrote down are necessary, but not sufficient conditions.

In the regime where the fluctuations are small with respect to the averages (requiring $\mathfrak{h}_{i}^{\prime} \ll 1$ ), we can take the average of the Hamiltonian constraint (12) and of the new constraint (16), and with simple algebra make further progress. In this approximation:

$$
\begin{gathered}
\mathfrak{h}_{1}^{\prime} \approx \frac{l_{P}^{2}}{3 V_{c} a^{3} H} \\
\mathfrak{h}_{2}^{\prime} \approx \frac{l_{P}^{2}}{3 V_{c} \tau_{C S} \Lambda^{-1}},
\end{gathered}
$$

where all the variables on the RHS are averages (corresponding to their classical values), subject to $H^{2}=\frac{\kappa}{6} \rho+\frac{\Lambda}{3}-\frac{k}{a^{2}}$ and $\tau_{C S}=H a\left(H^{2} a^{2}+3 k\right)$ (this is just a rewrite of Eq. (12) including matter, and of (16)). The first equation can be written as:

$$
1=\Omega_{\rho}+\Omega_{k}+\Omega_{\Lambda}
$$

with the usual definitions for $\Omega_{i}$, so that:

$$
\frac{\mathfrak{h}_{2}}{\mathfrak{h}_{1}} \approx \frac{3 \Omega_{\Lambda}}{1-3 \Omega_{k}} .
$$

Hence, even in the (semi-)classical regime, the two Planck parameters can be very different: if matter dominates $\Lambda$, for example, or if curvature is appreciable (such as in the "crisis" scenario of [19], but see [20]). It may be possible for $a$ and $b$ to behave classically for all purposes whereas the fluctuations in $\Lambda$ are still non-negligible. This could be an interesting interface with the issues raised by [22].

\section{More Speculative Examples}

The examples considered so far were derived from first principles, starting from a classic dynamics and following standard quantization methods. But we can be more speculative and entertain more general ad hoc Planck parameters [23]. A 
relevant example for this paper can be found in [24]. This differs from the cases investigated here so far in two ways. Firstly, an arbitrary function of $\Lambda$ (not necessarily $\Lambda^{-1}$, as in Eq. (18)) was proposed as the conjugate to the CS time, $\tau_{C S}$. Secondly (and more importantly), the Planck parameter postulated in [24] does not depend on $V_{c}$, in contrast with (6). This has the extreme effect of delocalizing the universe in time on all scales, when it becomes evident that we live in a sharp state of the cosmological constant, strongly peaked around zero. Thus, in [24], the Planck parameter pertaining to two intensive quantities ( $\Lambda$ and CS time) is itself an intensive quantity.

We could also consider intermediate situations. The reason why a $1 / V_{c}$ factor appears in the expressions for $\mathfrak{h}^{\prime}$ derived from first principles is that the starting point is an action which is extensive. Hence the canonical conjugate of an intensive variable in MSS must be extensive, but if we ignore the volume factor in the resulting observable within MSS (for example caring only about the Hubble parameter, or the CS time), then the factor of $V_{c}$ appears in the denominator on the right-hand side of the commutation relations, as we saw.

This is necessary, if building the quantum theory from the classical one; but by freeing ourselves from this constraint, we could envisage any other scaling. If we were to appeal to the holographic principle, for example, the factor of $V_{c}$ would be the area of the comoving region $A_{c} \sim l_{c}^{2}$, where $l_{c}$ is the linear dimension of the region under study. If we were instead to imagine super-extensive scalings, such as the one proposed in [25], a power of $l_{c}$ higher than 3 would replace $V_{c}$ :

$$
V_{c} \rightarrow l_{c}^{n}
$$

where we can relate $n$ to the exponent $\Delta$ defined in [25] according to $n=3\left(1+\frac{\Delta}{2}\right)$. If all the time dependent quantities can be related to the Hubble parameter, we would have a dimensionless Planck parameter of the form:

$$
\mathfrak{h}^{\prime} \sim \frac{l_{P}^{2}}{l^{n} H^{n-2}}
$$

where $l$ is the proper size of the region under examination. Hence the border between classical and quantum would now be located at scale:

$$
l_{Q} \sim\left(l_{P}^{2} H^{2-n}\right)^{1 / n} .
$$

instead of (11). The holographic example $(n=2)$ leads to the naive expectation $l_{Q} \sim l_{P}$. The unexpected result that the border between classical and quantum can be larger than $l_{P}$ results from the conservative assumption that the conjugate of an intensive quantity must be extensive. Super-extensive momenta would lead to larger and larger $l_{Q}$. The situation in [24] corresponds to the discontinuous $n \rightarrow \infty$ limit.

As a last speculative example, we may wonder whether our generalized definition of Planck parameter and of quantum uncertainty may affect thermodynamical quantities under extreme gravitational situations. For example, it could be that thermodynamic conjugates, such as the temperature $T$ and entropy $S$, are subject to uncertainty relations: 


$$
\sigma(S) \sigma(T) \geq \mathfrak{h}
$$

with $\mathfrak{h}$ scaling with the volume and temperature in generic ways. In quantum gravity motivated situations, we can expect $l_{P}$ to appear in its expression for $\mathfrak{h}$ but otherwise, it could take any form.

\section{Discussion}

We remark the important anthropic implications of allowing for a variable Planck parameter $[1,26]$. The non-relativistic Schrödinger equation for atomic physics possesses a simple scaling property. By a suitable scaling of variables it can be written in a form in which no constants of Nature appear [1, 27]. Considering a situation with two different sets of constants (primed and unprimed), if $E$ was the original energy eigenstate and $E^{\prime}$ is the new one, then

$$
\frac{\hbar^{\prime 2} E^{\prime}}{e^{\prime 4} m_{e}^{\prime}}=\frac{\hbar^{2} E}{e^{4} m_{e}} .
$$

where $e$ and $m_{e}$ are the electron's charge and mass. Therefore, if an atom exists as a solution of the equation with the unprimed constants of Nature, then a corresponding atom will exist with constants given by the primed variables.

We see that large changes of $\hbar$ into what we think of as the classical regime still allows atoms to exist: larger $\hbar$ means larger atoms. All the unusual properties of atomic systems, like water and DNA, do not depend on these constants if there are simultaneous variations of the other constants in the scaling. They just depend on the geometric factors like $2 \pi$. The uncertainty principle will be made of $\Delta p \sim p \sim m_{e} c$ and $\Delta x \sim x$ in the $x$ coordinates, so

$$
\Delta p \Delta x \sim m_{e} c x \gtrsim \hbar
$$

whereas in the ' system we would have $\Delta p^{\prime} \sim p^{\prime} \sim m_{e}^{\prime} c^{\prime}$ and $\Delta x^{\prime} \sim x^{\prime}$ so

$$
\Delta p^{\prime} \Delta x^{\prime} \sim m_{e}^{\prime} c^{\prime} x^{\prime} \gtrsim \hbar^{\prime}
$$

For further discussion of the implications the reader is referred to [1].

To conclude, we have examined situations where a contextual, time and sizedependent Planck parameter can be defined. At first, we worked in QC from first principles, deriving such a size and time dependent quantity, which can also depend on the pair of variables under study. We used this contextual Planck parameter to define the classical limit of QC, concluding that, even in the most standard theory, classicality depends on the comoving region's size, the time and the variables under study. In the standard QC set up, where one studies a whole closed Universe, there is a single "time" for the universe to pop up out of the quantum epoch, possibly after creation ex nihilo. By introducing a space scale into the problem, we have shown that at any time after the Planck epoch, there are always regions that remain quantum. These must only be smaller than $l_{P}$ at Planck time: later on, they must in fact 
be smaller than $l_{Q}=\left(l_{P}^{2} H^{-1}\right)^{1 / 3}$. As $H$ decreases in time, $l_{Q}$ increases, so that there is always a scale larger than $l_{P}$ where the quantum fluctuations are appreciable.

In theories where the cosmological constant, $\Lambda$, is dynamical it is possible for the latter to remain quantum even in contexts where everything else is deemed classical. Could this assist in our understanding of the cosmological constant problem?

We thank S. Alexander and D. Jennings for discussions related to this paper. JM was funded by the STFC Consolidated Grant ST/L00044X/1. JDB was funded by STFC

Open Access This article is licensed under a Creative Commons Attribution 4.0 International License, which permits use, sharing, adaptation, distribution and reproduction in any medium or format, as long as you give appropriate credit to the original author(s) and the source, provide a link to the Creative Commons licence, and indicate if changes were made. The images or other third party material in this article are included in the article's Creative Commons licence, unless indicated otherwise in a credit line to the material. If material is not included in the article's Creative Commons licence and your intended use is not permitted by statutory regulation or exceeds the permitted use, you will need to obtain permission directly from the copyright holder. To view a copy of this licence, visit http://creativecommons.org/ licenses/by/4.0/.

\section{References}

1. Barrow, J.D., Tipler, F.J.: The Anthropic Cosmological Principle. Oxford University Press, Oxford, (1988). ISBN 978-0-19-282147-8

2. Mangano, G., Lizzi, F., Porzio, A.: Inconstant Planck's constant. Int. J. Mod. Phys. A 30(34), 1550209 (2015). https://doi.org/10.1142/S0217751X15502097

3. Budiyono, A., Dipojono, H.K.: Nonlinear Schrödinger equations and generalized Heisenberg uncertainty principle from estimation schemes violating the principle of estimation independence. Phys. Rev. A 102(1), 012205 (2020)

4. Budiyono, A.: No-signaling non-unitary modification of quantum dynamics within a deterministic model of quantization. Ann. Phys. 347, 170-191 (2014)

5. Heisenberg, W.: Uber den anschaulichen Inhalt der quantentheoretischen Kinematik und Mechanik. Z. Phys 43, 172-198 (1927). https://doi.org/10.1007/BF01397280

6. Schrodinger, E.: About Heisenberg uncertainty relation. Bulg. J. Phys. 26, 193 (1999)

7. Poulin, D., Yard, J.: New J. Phys. 9, 156 (2007)

8. Jennings, D., Leifer, M.: Contemporary Physics Vol. 56 (2015); arXiv:1501.03202

9. Vilenkin, A.: Approaches to quantum cosmology. Phys. Rev. D 50, 2581-2594 (1994). https://doi. org/10.1103/PhysRevD.50.2581

10. Magueijo, J., Zlosnik, T., Speziale, S.: Quantum cosmology of a dynamical Lambda. arXiv: 2006.05766

11. Halliwell, J.J.: Decoherence in quantum cosmology. Phys. Rev. D 39, 2912 (1989). https://doi. org/10.1103/PhysRevD.39.2912

12. Unruh, W.G., Zurek, W.H.: Reduction of a wave packet in quantum Brownian motion. Phys. Rev. D 40, 1071 (1989). https://doi.org/10.1103/PhysRevD.40.1071

13. Habib, S.: The classical limit in quantum cosmology. 1 Quantum mechanics and the Wigner function. Phys. Rev. D 42, 2566-2576 (1990). https://doi.org/10.1103/PhysRevD.42.2566

14. Alexander, S., Cortês, M., Liddle, A.R., Magueijo, J., Sims, R., Smolin, L.: Zero-parameter extension of general relativity with a varying cosmological constant. Phys. Rev. D 100(8), 083506 (2019a). https://doi.org/10.1103/PhysRevD.100.083506

15. Alexander, S., Cortês, M., Liddle, A.R., Magueijo, J., Sims, R., Smolin, L.: Cosmology of minimal varying Lambda theories. Phys. Rev. D 100(8), 083507 (2019b). https://doi.org/10.1103/PhysR evD.100.083507 
16. Lazar, M., Hehl, F.W.: Cartan's spiral staircase in physics and in particular, in the gauge theory of dislocations. Found. Phys. 40, 1298-1325 (2010). https://doi.org/10.1007/s10701-010-9440-4

17. Magueijo, J., Złośnik, T.: Parity violating Friedmann Universes. Phys. Rev. D 100(8), 084036 (2019). https://doi.org/10.1103/PhysRevD.100.084036

18. Smolin, L., Soo, C.: The Chern-Simons invariant as the natural time variable for classical and quantum cosmology. Nucl. Phys. B 449, 289-316 (1995). https://doi.org/10.1016/0550-3213(95)00222 $-\mathrm{E}$

19. Di Valentino, E., Melchiorri, A., Silk, J.: Planck evidence for a closed Universe and a possible crisis for cosmology. Nat. Astron. 4(2), 196-203 (2019). https://doi.org/10.1038/s41550-019-0906-9

20. Handley, W.: arXiv:1908.09139

21. Randono, A.: A mesoscopic quantum gravity effect. Gen. Rel. Grav. 42, 1909-1917 (2010) https:// doi.org/10.1007/s10714-010-0982-8[arXiv:0805.2955 [gr-qc]]

22. Wang, Q., Zhu, Z., Unruh, W.G.: How the huge energy of quantum vacuum gravitates to drive the slow accelerating expansion of the universe. Phys. Rev. D 95, 103504 (2017). https://doi. org/10.1103/PhysRevD.95.103504

23. Barrow, J.D., Magueijo, J.: Do we live in an eigenstate of the fundamental constants operators? Phys. Rev. D 99(2), 023509 (2019). https://doi.org/10.1103/PhysRevD.99.023509

24. Magueijo, J., Smolin, L.: A Universe that does not know the time. Universe 5, 84 (2019). https://doi. org/10.3390/universe5030084

25. Barrow, J.D.: The area of a rough Black hole. arXiv: 2004.09444

26. Lieb, E.H.: The stability of matter. Rev. Mod. Phys. 48, 553-569 (1976). https://doi.org/10.1103/ RevModPhys.48.553

27. Hartree, D.R.: The Wave Mechanics of an Atom with a Non-Coulomb Central Field. Part I. Theory and Methods. (1928)

Publisher's Note Springer Nature remains neutral with regard to jurisdictional claims in published maps and institutional affiliations. 